\title{
Evaluación de la Contaminación Sonora en la Ciudad de Tacna
}

RESPONSABLE: MSc. Urbano Humberto Pérez Rudas †

\begin{abstract}
RESUMEN La determinación del presente estudio es conocer y evaluar cualitativa y cuantitativamente los niveles de ruido generados por los establecimientos que se dedican a las actividades rutinarias en el área urbana de la ciudad de Tacna, para lo cual se ha requerido realizar las mediciones con un sonómetro tipo 1 , de los niveles de ruido generados por dichos establecimientos, lo que permitirá adicionalmente evaluar el cumplimiento que hacen del D.S. $N^{o} 085$ 2003-PCM, del 24/10/2003, Reglamento de Estándares Nacionales de Calidad Ambiental para Ruido
\end{abstract}

Se tomaron muestras de 6 puntos estratégicos de la ciudad, en diferentes horarios y dias de la semana, teniendo en consideración los niveles de tráfico, las variaciones por el estado atmosférico y climatológico durante el periodo de medida, concluyendo en la elaboración de un mapa sonoro preliminar con mediciones de corta duración en el microcentro de la ciudad, asi como los resultados del monitoreo piloteados en el mapa de ruidos de zonas de mayor impacto sonoro.
MIEmbros: Msc. Julio Fernández Prado

\begin{abstract}
The determination of this study is to evaluate qualitatively and quantitatively and the noise levels generated by establishments engaged in routine activities in the urban area of the city of Tacna, for which it has been required to perform measurements with a sound level meter type 1 , noise levels generated by these establishments, which will further evaluate the performance that make the DS No. 085-2003PCM, of 24/10/2003, Regulation National Environmental Quality Standards for Noise
\end{abstract}

He took samples of 6 strategic points in the city at different times and days of the week taking into account traffic levels, variations in atmospheric and climate state during the measurement period. Conclusion in the preparation of a preliminary map noise measurements of short duration in the micro downtown and monitoring results plotted on the map noise greater noise impact zones.
INTRODUCCIÓN. El ruido es el contaminante más común y puede definirse como cualquier sonido que sea calificado por quien lo recibe como algo molesto, indeseado, inoportuno, perturbador o desagradable. Los principales efectos del ruido se han considerado como auditivos y no auditivos; los efectos auditivos están en correlación con la pérdida de la capacidad auditiva de las personas expuestas (el daño auditivo no solo depende de su nivel, sino de su duración, se acepta que un medio ambiente sonoro por debajo de $75 \mathrm{~dB}$ no es dañino para la salud auditiva) y los no auditivos son los que pueden generar estrés por perturbar el sueño, por ser transformadores de las actividades humanas cotidianas.

Una ciudad ruidosa nace por generación espontánea cuando no se han tenido en cuenta los problemas de ruido urbano en el planeamiento. Ello repercute en el transeúnte, que no logra mantener una conversación normal en las aceras, balcones y espacios exteriores, en la ciudad, pero también interviene en la arquitectura, que debe utilizar sofisticadas técnicas de aislamiento para proteger a sus moradores.

Un Mapa Sonoro de una ciudad, pretende ser una herramienta de planeamiento urbano; es decir, facilita una información sobre el estado de la ciudad en materia de contaminación ambiental por ruido que, combinada con el resto de información sobre el entorno urbano, debe ser integrada dentro de la gestión general de la ciudad para conseguir una calidad de vida adecuada a la población.

La ciudad de Tacna presenta una intermedia contaminación sonora, proveniente básicamente del auge comercial y urbanístico, así como la densidad del tráfico vehicular que circula sobre las principales vías que atraviesan las diferentes calles o avenidas en las que está asentado el comercio.

\section{MATERIALY MÉTODO}

El ruido es cualquier sonido calificado, no deseado, que molesta, perjudica o afecta a las personas o su salud y que puede producir efectos fisiológicos, psicológicos y sociales no deseados.

Se llama contaminación acústica a la que altera las condiciones de sonidos normales en una determinada zona, es aquella que se genera por un sonido no deseado, que afecta negativamente a la calidad de vida y sobre todo a aquellos individuos que desarrollan actividades industriales.

Las causas fundamentales de la contaminación acústica son el aumento espectacular del parque automovilístico, las actividades industriales, las obras públicas y la construcción, los servicios de limpieza y de 
recogida de basura, sirenas y alarmas, así como las actividades lúdicas y recreativas.

\section{Marco Legal Nacional}

Las regulaciones implican la disminución de los niveles de ruido generados por las actividades humanas. El objetivo de la disminución de los niveles de ruido es proteger la salud y el bienestar de la población. Para lograr este objetivo es necesario aplicar todas las medidas posibles en el control del ruido como: prevenir y mitigar emisiones, aplicar tecnología de bajo nivel de ruido, restricciones temporales para las actividades que son indebidamente ruidosas. Los efectos del ruido sobre la salud se están generalizando. Por ello, es esencial tomar acciones para limitar y controlar la exposición al ruido ambiental, teniendo en cuenta que a un nivel de presión de sonido de $65 \mathrm{~dB}$, el acto de conciliar el sueño puede ser perturbado enormemente produciendo un estado de enojo en las personas. Queda claro que el ruido comunitario es un problema de salud ambiental genuino.

Estándares Nacionales de Calidad Ambiental para Ruido

\begin{tabular}{|l|c|c|}
\hline \multirow{2}{*}{$\begin{array}{c}\text { Zonas de } \\
\text { Aplicación }\end{array}$} & \multicolumn{2}{|c|}{$\begin{array}{c}\text { Valores Expresados en } \\
\text { LAeqT }\end{array}$} \\
\cline { 2 - 3 } $\begin{array}{c}\text { Zorario } \\
\text { Zona de Protección } \\
\text { Especial }\end{array}$ & $\begin{array}{c}\text { Horario } \\
\text { Nocturno }\end{array}$ \\
\hline Zona Residencial & 50 & 40 \\
\hline $\begin{array}{l}\text { Zona Comercial } \\
\text { Zona Industrial }\end{array}$ & 60 & 50 \\
\hline
\end{tabular}

\section{Clasificación del Ruido Según su Variación}

El ruido según su variación se clasifica de la siguiente forma:

- Ruido Constante:

- Ruido Fluctuante:

- Ruido Intermitente

- Ruido Impulsivo:

\section{Efectos Adversos del Ruido Sobre la Salud}

Las consecuencias de la contaminación acústica para la salud las podemos describir según sus efectos adversos en:

- Efectos sobre la audición,

- Efectos sobre el sueño,

- Efectos sobre las funciones fisiológicas,

- Efectos sobre la salud mental,

- Efectos sobre el rendimiento,

- Efectos sociales y sobre la conducta. La molestia del ruido,

- Efectos combinados del ruido de fuentes mixtas sobre la salud,
Subgrupos vulnerables.

Equipo Empleado para Medir los Ruidos.

Sonómetros. El medidor de presión sonora, conocido como sonómetro o también como decibelimetro, es el instrumento para las mediciones acústicas más simple y está diseñado para determinar el nivel sonoro con intercalación de unos adecuados circuitos de ponderación de frecuencias. Un medidor de nivel sonoro debe cumplir con las especificaciones de las Normas IEC 651 - IEC 804 o con la Norma ANSI S1.4

\section{Resultados de las Mediciones Efectuadas, Análisis y Discusión de los Resultados de las Mediciones}

Los resultados de las mediciones efectuadas en horario diurno y nocturno, de los niveles de ruido ambiental generados están representados en el plano sonoro de Tacna. Se presentan en el cuadro siguiente:

\begin{tabular}{|c|c|c|c|c|}
\hline & \multirow{2}{*}{ ACTIVIDADES } & \multirow{2}{*}{$\begin{array}{l}\text { NÚMERO } \\
\text { MUESTRAS }\end{array}$} & \multicolumn{2}{|c|}{$\begin{array}{l}\text { PROMEDIO NIVELES } \\
\text { RUIDO DB. }\end{array}$} \\
\hline & & & Máximo & MíNIMo \\
\hline 1 & Mercadillo Bolognesi & 3 & 82,98 & 77,15 \\
\hline 2 & Mercadillo Polvos Rosados & 3 & 83,39 & 77,72 \\
\hline 3 & Mercadillo Tüpac Amaru & 3 & 81,90 & 77,57 \\
\hline 4 & Esquina del Movimiento & 3 & 80,33 & 74,87 \\
\hline 5 & 2 de Mayo & 3 & 82,17 & 76.71 \\
\hline 6 & Mercado Central & 3 & 85,48 & 78,66 \\
\hline 7 & Correo Central & 3 & 84,00 & 78,66 \\
\hline
\end{tabular}

La actividad en el Mercado Central es la que presenta el nivel de ruido más alto $85,48 \mathrm{db}$, y la de Esquina del Movimiento el nivel de ruido más bajo $80,33 \mathrm{db}$.

Los detalles de las mediciones realizadas se encuentran representadas en los cuadros adjuntos.

\section{Resultados de las Mediciones Efectuadas por Actividad}

\section{Medición del Nivel de Ruido Ambiental en el Mercadillo} Bolognesi

Análisis

En el frontis del Mercadillo Bolognesi, especialmente en la Av. Coronel Mendoza, se tomó la mayor parte de las muestras, estando en relación directa con las fuentes de emisión del ruido generado por las diferentes actividades del entorno, dando por resultado niveles de ruido altos y constantes.

Del cuadro se puede observar que las operaciones de la empresa monitoreada obtuvieron niveles de ruido por encima de los Estándares de Calidad de Ruido según el D.S. No 085-2003-PCM - Reglamento de Estándares Nacionales de Calidad Ambiental para Ruido. 
Medición del Nivel de Ruido Ambiental en Mercadillo Polvos Rosados

Análisis

El ruido medido en ambientes a exteriores del Mercadillo provoca molestias al vecindario, debido a que las fuentes ruidosas se encuentran alejadas de su perímetro y la señal que llega a su lindero no es significativa a comparación del tráfico que circula alrededor de la misma.

En las mediciones realizadas se determinó que los niveles de ruido medidos en el exterior del Mercadillo (ruido ambiental) son superiores a los fijados en el D.S. $\mathrm{N}^{\text {o }}$ 085-2003-PCM - Reglamento de Estándares Nacionales de Calidad Ambiental para Ruido.

\section{Medicion del Nivel de Ruido Ambiental en Mercadillo Tupac Amaru}

Análisis

Se observa que los ruidos generados en el exterior del Mercadillo, tienen niveles altos, y que estos niveles de ruidos se ven incrementados por diversas actividades que se realizan en el frontis y acera de Túpac Amaru, además por el tráfico de vehículos livianos.

En las instalaciones del Mercadillo Túpac Amaru, se realizaron las mediciones en dos puntos importantes en cada una de ellas, que están en relación directa con las fuentes de emisión del ruido generado por ellas, dando por resultado niveles de ruido altos y constantes.

En las mediciones realizadas en la muestra se determinó que los niveles de ruido medidos en el exterior del Mercadillo (ruido ambiental), son superiores a los fijados en el D.S. N ${ }^{\circ}$ 085-2003-PCM Reglamento de Estándares Nacionales de Calidad Ambiental para Ruido.

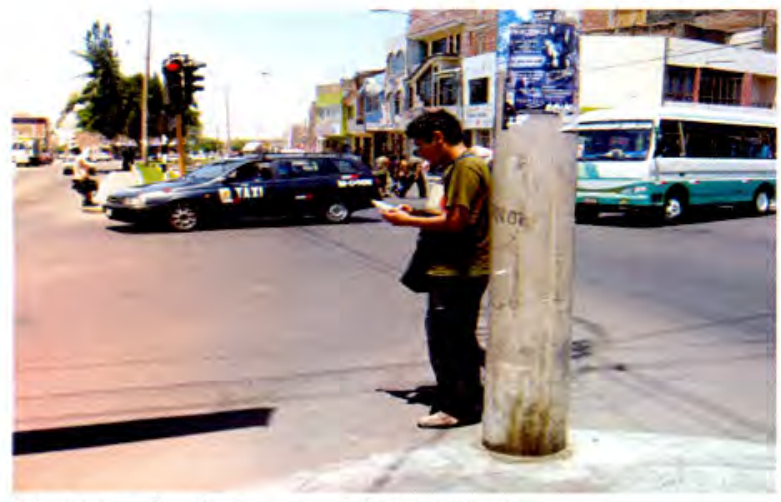

Monitoreo Ruido Esquina del Movimiento

Medicion del Nivel de Ruido Ambiental en la Esquina del Movimiento

Análisis

En las mediciones realizadas en la muestra se determinó que los niveles de ruido (ruido ambiental) medidos en el lugar denominado Esquina del
Movimiento son superiores a los fijados en el D.S. $\mathrm{N}^{\circ}$ 085-2003-PCM - Reglamento de Estándares Nacionales de Calidad Ambiental para Ruido.

Medicion del Nivel de Ruido Ambiental en el Mercado 2 de Mayo

Análisis

En el monitoreo del Mercado 2 de Mayo se observó que los niveles de ruido medidos en su exterior son ocasionados por diversas fuentes ajenas a dicho mercado, como es el tráfico vehicular existente entre las dos avenidas cercanas. En diferentes puntos se realizaron las mediciones y cada una de ellas se encuentra en relación directa con las fuentes de emisión del ruido generado por tales avenidas, dando por resultado niveles de ruido altos con picos elevados en hora punta del tráfico vehicular.

\section{Medición del Nivel de Ruido Ambiental en el Mercado Central}

Análisis

Las mediciones realizadas en la muestra permiten determinar que los niveles de ruido son superiores a los fijados en el D.S. N ${ }^{\circ}$ 085-2003-PCM - Reglamento de Estándares Nacionales de Calidad Ambiental para Ruido.

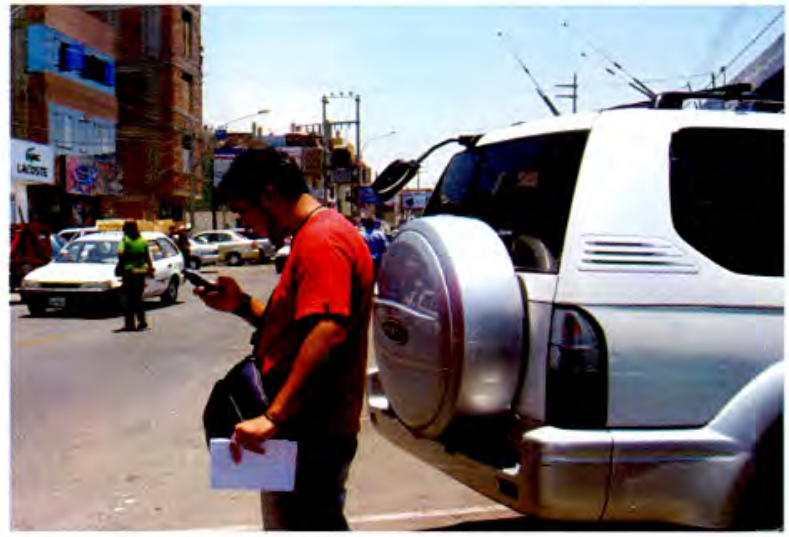

Monitoreo Ruido Mercadillo Bolognesi

Medición del Nivel de Ruido Ambiental en el Mercadillo Bolognesi

\begin{tabular}{|c|c|c|c|c|c|c|c|c|c|}
\hline \multicolumn{10}{|c|}{ MERCADILOOBOLOGNEST } \\
\hline \multirow[t]{2}{*}{$N^{\circ}$} & \multirow{2}{*}{ HORA } & \multicolumn{2}{|c|}{ PRIMERO } & \multicolumn{2}{|c|}{ SEGUNDO } & \multicolumn{2}{|c|}{ TERCERO } & \multicolumn{2}{|c|}{ PROMEDIOS } \\
\hline & & МÁXIMO & MINIMO & MAXXMM0 & MÍNIMO & мíxisyo & MiNIM0 & MixIM0 & MiNIMO \\
\hline 1 & $8: 30$ HRS & 59,60 & 55,10 & 7,90 & 70,30 & 85,90 & 80,50 & 74,47 & 68,63 \\
\hline 2 & $10: 00$ HRS & 75,00 & 70,00 & 79,70 & 75,80 & 79,70 & 75,30 & 7,13 & 73,70 \\
\hline 3 & 11.30 HRS & 77,20 & 73,00 & 83,30 & 78,00 & 85,10 & 80,10 & 81,87 & 77,03 \\
\hline 4 & 13:00 HRS & 80,00 & 73.00 & 8,10 & 79,00 & 85.10 & 79,00 & 83,40 & 77,00 \\
\hline 5 & $14: 30 \mathrm{HRS}$ & 88,40 & 82,00 & 70,00 & 65,60 & 83,30 & 79,40 & 80,57 & 75,67 \\
\hline 6 & 16.00 HRS & 79,10 & 73,00 & 75,40 & 70,00 & 79,10 & 73,00 & 77,87 & 72,00 \\
\hline 7 & $17: 30$ HRS & 81,40 & 78,40 & 92.50 & 83,50 & 88,40 & 82,00 & 87,43 & 81,30 \\
\hline 8 & $19.00 \mathrm{HRS}$ & 94,80 & 88,40 & 91,70 & 77,80 & 94,80 & 87,00 & 93,77 & 84,40 \\
\hline 9 & $20.30 \mathrm{HRS}$ & 92,50 & 85,80 & 89,50 & 85,00 & 85,90 & $83,0)$ & 89.30 & 84,60 \\
\hline & PROTOL & & & & & & & 82,98 & 7,15 \\
\hline
\end{tabular}


Medición del Nivel de Ruido Ambiental en el Mercadillo Polvos Rosados.

\begin{tabular}{|c|c|c|c|c|c|c|c|c|c|}
\hline \multicolumn{10}{|c|}{ MERCADILLO POLVOS ROSMDES } \\
\hline $\mathbb{N}^{\mathrm{P}}$ & HORA & \multicolumn{2}{|c|}{ PAAMERO } & \multicolumn{2}{|c|}{ SEANDO } & \multicolumn{2}{|c|}{ TFROFRO } & \multicolumn{2}{|c|}{ FROMETIOS } \\
\hline & & Mixmo & MíNMO & Mixrno & Mív'so & MixIMo & MíNINo & мíxiмo & MivMo \\
\hline & 08:45 HRS & 61,40 & 57,30 & 8090 & 75,00 & 92,10 & 87,40 & 78,13 & 73,2 \\
\hline & 10.15 HRS & 75,70 & 69,90 & 124,10 & 99,30 & 92,10 & 87,40 & 97,30 & 85,53 \\
\hline 3 & $11: 45 \mathrm{HRS}$ & 71,30 & 69,60 & 80,60 & 75,00 & 78,60 & 73,00 & 76,83 & 72,53 \\
\hline 4 & 13:15 HRS & 8,10 & 79,00 & 78,60 & 71,90 & 86,60 & 83,00 & $83, \pi$ & 77,97 \\
\hline 5 & 14:45 HRS & 91,40 & 85,00 & 84,00 & $\Leftrightarrow, 40$ & 0,60 & 75,30 & 85,33 & 76.57 \\
\hline 6 & 16.15 HRS & 7230 & 69,00 & 85,10 & 79,90 & 72,30 & $6 \rightarrow 50$ & 76.57 & 72,8 \\
\hline & $17: 45 \mathrm{HRS}$ & 76,80 & 70,50 & 73,20 & 83,90 & 91,40 & 85,00 & 80,47 & 79,80 \\
\hline & 19.15 HRS & 86,60 & 79,90 & 86,30 & 81,40 & 86,60 & 80,90 & 86,50 & 80,73 \\
\hline & $20.45 \mathrm{HISS}$ & 80,60 & 73,00 & 84,20 & 8030 & 92,10 & 87,60 & 85,63 & 80,30 \\
\hline & PROTIOL & & & & & & & 83,39 & 7,72 \\
\hline
\end{tabular}

\section{Medición del Nivel de Ruido Ambiental en Mercadillo Tupac Amaru}

\begin{tabular}{|c|c|c|c|c|c|c|c|c|c|}
\hline \multicolumn{10}{|c|}{ MERCADILO TUPAC AMARU } \\
\hline \multirow{2}{*}{$\mathrm{N}$} & \multirow{2}{*}{ HORA } & \multicolumn{2}{|c|}{ PRIMERO } & \multicolumn{2}{|c|}{ SEGUNDO } & \multicolumn{2}{|c|}{ TERCERO } & \multicolumn{2}{|c|}{ PROMEDIOS } \\
\hline & & MÁXIMO & мімммо & мíxiмo & MíNmo & Míxм10 & MiNimo & мixмmo & MÍNTMO \\
\hline 1 & $9.00 \mathrm{HRS}$ & 77,20 & 72,40 & 85,90 & 81,00 & 82,50 & 78,40 & 81.87 & 77.27 \\
\hline 2 & $10.30 \mathrm{HPS}$ & 74,50 & 70,10 & 88,60 & 83,00 & 88,60 & 80.50 & 8390 & 77.87 \\
\hline 3 & $1200 \mathrm{HRS}$ & 81,60 & 77,50 & 87,60 & 83,00 & 75,00 & 69,90 & 81,40 & 76.80 \\
\hline 4 & $13: 30 \mathrm{HIRS}$ & 83,90 & 77,00 & 75,00 & 68,90 & 83,30 & 80,10 & 80,73 & 75,33 \\
\hline 5 & 15.00 HRS & 82,80 & 78,90 & 73,90 & 69,30 & 87,60 & 83,00 & 81,43 & 77,07 \\
\hline 6 & $16: 30 \mathrm{HRS}$ & 72,30 & 69,00 & 79,80 & 74,90 & 74,10 & 70,00 & 75,40 & 71.30 \\
\hline 7 & $18.00 \mathrm{HRS}$ & 78,60 & 72,70 & 74,60 & 86,30 & 82,80 & 73,00 & 78.67 & 77,33 \\
\hline 8 & $19.30 \mathrm{HRS}$ & 83,30 & 77.50 & 92,30 & 86,50 & 83,30 & 84,00 & 86,30 & 82,67 \\
\hline 9 & $21: 00 \mathrm{HRS}$ & 85,00 & 79,40 & 94,60 & 88.40 & 82,50 & 79,60 & 87.37 & 82,47 \\
\hline & PRO/TOL & & & & & & & 81,90 & 7757 \\
\hline
\end{tabular}

Medición del Nivel de Ruido Ambiental en la Esquina del Movimiento

\begin{tabular}{|c|c|c|c|c|c|c|c|c|c|}
\hline \multicolumn{10}{|c|}{ ESOUINA DEL MONIMIENIO } \\
\hline \multirow[t]{2}{*}{$\mathrm{N}$} & \multirow[t]{2}{*}{ HORA } & \multicolumn{2}{|c|}{ RRIMERO } & \multicolumn{2}{|c|}{ SEGINDO } & \multicolumn{2}{|c|}{ TERCERO } & \multicolumn{2}{|c|}{ PROMEDIOS } \\
\hline & & Mixivo & мі́ммо & Мі́xIMo & MiNIno & MiNMo & MíNiмo & міхтмо & MÍNINKO \\
\hline 1 & $0830 \mathrm{HPS}$ & 78,60 & 71,50 & 78,00 & 71,00 & 8460 & 80,60 & 80,40 & 74,37 \\
\hline 2 & $1000 \mathrm{HPS}$ & 78,90 & 72,00 & 82,20 & 75,00 & 82,20 & 79,40 & 81,10 & 75,47 \\
\hline 3 & 11:30HRS & 87,80 & 82,60 & 54,80 & 44,00 & 84,00 & 79,00 & 75,53 & 68,53 \\
\hline 4 & $1300 \mathrm{HISS}$ & 80,40 & 76,00 & 84,00 & 79,30 & 79,40 & 6801 & 81,27 & 74,4 \\
\hline 5 & 1430 HIS & 92,90 & 86,30 & 78,30 & 72.10 & 54,80 & 50,00 & 75,33 & 69,47 \\
\hline 6 & 1600 HISS & 82.10 & 76,00 & 80,50 & 75,10 & 82,10 & 7930 & 81,57 & 76,80 \\
\hline 7 & 1730 HIS & 77,30 & 75,30 & 76,50 & 78,90 & 92,90 & 83,00 & 82,23 & 79,07 \\
\hline 8 & 1900 HRS & 83,40 & 78,20 & 86,80 & 75,60 & 83,40 & 84,90 & 84,53 & 79,57 \\
\hline 9 & $2030 \mathrm{HRS}$ & 77,70 & 71.50 & 80,70 & 77,40 & 84,60 & 79,60 & 81.00 & 76,17 \\
\hline & FROTLL. & & & & & & & 80,33 & 7487 \\
\hline
\end{tabular}

Medición del Nivel de Ruido Ambiental en el Mercado 2 de Mayo

\begin{tabular}{|c|c|c|c|c|c|c|c|c|c|}
\hline \multirow{3}{*}{ so } & \multicolumn{9}{|c|}{2 DE MAYO } \\
\hline & HORA & PRIS & ERO & SEG & NDO & TERC & ERO & PROM & DIOS \\
\hline & & мíximo & мімімо & міхтмо & MINIMO & iximo & Mivimo & мiximo & Mivino \\
\hline 1 & 08:45 HRS & 71,50 & 67,30 & 88,00 & 82,00 & 83,40 & 79,70 & 80,97 & 76,33 \\
\hline 2 & \begin{tabular}{|l|}
$10: 15 \mathrm{HRS}$ \\
\end{tabular} & 77,00 & 71,00 & 89,50 & 80.40 & 89,50 & 85,40 & 85,33 & 78,93 \\
\hline 3 & 11:45 HRS & 85,60 & 69,40 & 79,30 & 72,00 & 86,10 & 82,10 & 83,67 & 74,50 \\
\hline 4 & 13:15 HRS & 89,60 & 82,00 & 86,10 & 80,30 & 71,20 & 6750 & 82,30 & 76,60 \\
\hline 5 & 14:45 HRS & 80,01 & 79.90 & 80,00 & 76,10 & 79,30 & 72,00 & 79,77 & 76,00 \\
\hline 6 & 16:15 HRS & 90,20 & 84,00 & 83,60 & 78,50 & 90,20 & 85,60 & $88,(60)$ & 82,70 \\
\hline 7 & 17:45 HRS & 81,00 & 77,40 & 84,40 & 76,30 & 80,10 & 74,00 & 81,83 & 75,90 \\
\hline 8 & 19:15 HRS & 71.20 & 68,30 & 81,10 & 78,70 & 71.20 & 69,40 & 74,50 & 72,13 \\
\hline 9 & $20: 45$ HRS & 85,00 & 80,10 & 81,20 & 73.60 & 83.40 & 78,10 & 83.20 & 77,27 \\
\hline & PRO/TOL & & & & & & & 82,17 & 76,71 \\
\hline
\end{tabular}

\section{Medición del Nivel de Ruido Ambiental en el Mercado} Central

\begin{tabular}{|c|c|c|c|c|c|c|c|c|c|}
\hline \multicolumn{10}{|c|}{ MERCADO CENTRAL } \\
\hline \multirow[t]{2}{*}{$\mathrm{N}^{\circ}$} & \multirow[t]{2}{*}{ HORA } & \multicolumn{2}{|c|}{ PRIMERO } & \multicolumn{2}{|c|}{ SEGUNDO } & \multicolumn{2}{|c|}{ TERCERO } & \multicolumn{2}{|c|}{ PROMEDIOS } \\
\hline & & ма́хІмо & MINIMO & MAXIMO & міхімо & MAXтMO & міхтмо & Mіхтмо & мinimo \\
\hline 1 & $09.00 \mathrm{HRS}$ & 80,60 & 55,10 & 80,00 & 76,00 & 88,80 & 82,50 & 83,13 & 71.20 \\
\hline 2 & $1030 \mathrm{HRS}$ & 94,00 & 87,00 & 92,50 & 88,10 & 92,50 & 88,00 & 93,00 & 87,70 \\
\hline 3 & $1200 \mathrm{HRS}$ & 82,80 & 77,80 & 72,70 & 66,00 & 80,90 & 75,00 & 78.80 & 72,93 \\
\hline & 13:30 HRS & 87,60 & 81,30 & 80,90 & 73,70 & 88,30 & 77,90 & 85,60 & 77,63 \\
\hline 5 & $15: 00$ HRS & 87,70 & 81,00 & 78,40 & 73,20 & 88,20 & 69.60 & 84,77 & 74,60 \\
\hline 6 & $16.30 \mathrm{HRS}$ & 87,90 & 79,90 & 85,00 & 80,10 & 84,60 & 80,50 & 85,83 & 80,17 \\
\hline 7 & 1800 HRS & 81,30 & 78,40 & 80,00 & 77,70 & 87,70 & 81,00 & 83,00 & 79,03 \\
\hline 8 & 19.30 HRS & 88,30 & 83,10 & 91,20 & 86,30 & 88,30 & 82,00 & 89,27 & 83,80 \\
\hline 9 & $21.00 \mathrm{HRS}$ & 82,60 & 78,20 & 86,30 & 80,90 & 88,80 & 83,60 & 85,90 & 80,90 \\
\hline & & & & & & & & 85,48 & 78,56 \\
\hline
\end{tabular}

Medición del Nivel de Ruido Ambiental en el Correo Central.

\begin{tabular}{|c|c|c|c|c|c|c|c|c|c|}
\hline \multicolumn{10}{|c|}{ CORREO CENTRAL } \\
\hline \multirow[t]{2}{*}{$N^{*}$} & \multirow[t]{2}{*}{ HORA } & \multicolumn{2}{|c|}{ PRIMERO } & \multicolumn{2}{|c|}{ SEGUNDO } & \multicolumn{2}{|c|}{ TERCERO } & \multicolumn{2}{|c|}{ PROMEDIOS } \\
\hline & & михммо & Misimo & Miximo & мivimo & MAXIMO & Misiмo & Miximo & NiNimo \\
\hline 1 & $08: 30 \mathrm{HRS}$ & 74,40 & 67,90 & 77,90 & 71,90 & 88,20 & 86,70 & 80,17 & 75,50 \\
\hline 2 & 10:00 HRS & 80,00 & 72,00 & 81,20 & 76,00 & 81,20 & 75,90 & 80,80 & 74,63 \\
\hline 3 & 11:30h RS & 87,90 & 83,00 & 82,90 & 73,00 & 88,00 & 82,00 & 86,27 & 79,33 \\
\hline 4 & 13:00 HRS & 91.70 & 86,30 & 88.00 & 83,20 & 83,70 & 79.50 & 87,80 & 83,00 \\
\hline 5 & 14:30 HRS & 88,20 & 83,00 & 81,40 & 76,20 & 82,90 & 76,90 & 84.17 & 78,70 \\
\hline 6 & $16: 00 \mathrm{HRS}$ & 93,70 & 87,40 & 82,70 & 79,30 & 93,70 & 90,70 & 90,03 & 85.80 \\
\hline 7 & 17:30 HRS & 82,40 & 78,80 & 85,90 & 72,60 & 88,20 & 81,90 & 85,50 & 77,77 \\
\hline 8 & 19:00 HRS & 83,70 & 78.10 & 95,30 & 83,10 & 83,70 & 79,30 & 87,57 & 80,17 \\
\hline 9 & 20:30 HRS & 77,90 & 71,90 & 88,00 & 82,40 & 88.20 & 83,00 & 84,70 & 79,10 \\
\hline & PRO/TOL & & & & & & & 85,22 & 79,33 \\
\hline
\end{tabular}

\section{CONCLUSIONES}

1.Se han efectuado las mediciones de los niveles de ruido generados por las diferentes actividades que se realizan frente a los centros comerciales en la ciudad de Tacna, lo que permite concluir que en todos los puntos de monitoreo han sobrepasado los niveles establecidos en el D.S. N ${ }^{\circ}$ 085-2003-PCM, del 24/10/2003, Reglamento de Estándares Nacionales de Calidad Ambiental para Ruido.

2.Del análisis de los niveles máximos de ruido ambiental medidos en horario diurno, tarde y nocturno, generados en el departamento de Tacna, por el funcionamiento de los establecimientos que operan en las actividades comerciales y mercados de abastos, se aprecia que en muchos casos son superiores a los $70 \mathrm{db}$ fijados para zonas comerciales en horario diurno, y en horario nocturno $60 \mathrm{db}$, según el Decreto Supremo No 085-2003PCM - Reglamento de Estándares Nacionales de Calidad Ambiental para Ruido.

3.Es de singular importancia la educación y concienciación, realizada por diversas vías, especialmente a través de programas de preservación de la salud.

4.El presente trabajo de investigación se realizó en una fase inicial, no tocando muchas variables que si darían mayores elementos para diseñar una alternativa eficiente de solución, especialmente en formular los Limites Máximos Permisibles de Ruidos para estas actividades.

5.Los mapas obtenidos de los diferentes puntos de monitoreo se representaron mediante un sistema de información geográfico, con el fin de determinar estado del ambiente sonoro de su entorno.

\section{REFERENCIAS BIBLIOGRÁFICAS}

GÓMEZ-Senent, E.; Santamarina, J.C.; Chiner, M.; Aragonés, P...The Design Dimentions. A Design Theory Proposal. 11 th ICED. Agosto 1997. Tampere, Finlandia. GÓMEZ-SENENT MARTÍNEZ, Eliseo. El proyecto Diseño en la Ingeniería. Servicio de publicaciones de la U.P.V. 1997.(R1).

Miguel BOLÍVAR JIMÉNEZ. Los Niveles De Ruido en las Actividades de Hidrocarburos en el Departamento le Lima. Tesis Maestría, 2007.

SANTOS DE LA CRUZ, Eulogio."Contaminación sonora por ruido vehicular en la avenida Javier Prado". Ind. data, ene./jun. 2007, vol.10, no.1, p.11-15. ISSN. 